\title{
Incidência de leishmaniose tegumentar americana no norte de Mato Grosso entre 2001 e 2008
}

\author{
Evaldir de Souza NOBRES ${ }^{1}$, Laudenice Aparecida de SOUZA², Domingos de Jesus RODRIGUES ${ }^{3}$
}

\begin{abstract}
RESUMO
A incidência da Leishmaniose Tegumentar Americana registrada no Escritório Regional de Saúde de Sinop o qual abrangem 17 municípios foi analisada considerando os números de casos notificados por município, sexo, faixa etária e sazonalidade. A coleta das informaçóes foi obtida no Sistema Nacional de Agravos de Notificação entre 2001 e 2008. Foi calculada a incidência da doença em cada município. Para avaliar a influência da sazonalidade sobre a LTA, foram usados os dados de janeiro de 2001 a dezembro de 2008. Os coeficientes de detecçáo para os anos de 2001 a 2008 foram maiores nos municípios de Nova Ubiratá (857,2 por 100.000 habitantes), Feliz Natal (834,5 por 100.000 habitantes) e Vera (557,0 por 100.000 habitantes). A média de incidência (334,9 por 100.000 habitantes) da ERS-Sinop foi 4,5 vezes maior que a média da regiáo norte. A faixa etária mais acometida foi entre 20 e 49 anos em ambos os sexos e, sendo que o sexo masculino foi o mais acometido $64,78 \%$. $\mathrm{O}$ número de casos registrados no período da seca foi de $60 \%$. Grande parte da população da região está exposta ao contato direto com o vetor da Leishmania, principalmente devido a alguns tipos de atividades econômicas desenvolvidas na regiáo tais como: $\mathrm{O}$ corte seletivo de madeira, a agropecuária e a mineração.
\end{abstract}

PALAVRAS-CHAVE: Epidemiologia, Saúde Pública, Prevalência, Amazônia.

\section{Incidence of cutaneous leishmaniasis in the north of Mato Grosso among 2001 and 2008}

\begin{abstract}
Incidence of cutaneous leishmaniasis recorded in the Health Regional Office of Sinop (ERS-SINOP), which covers 17 cities located at the north of Mato Grosso was examined considering the numbers of reported cases by municipalities, gender, age and rainfall seasonality. Data were obtained from the National System of Notification Diseases (Sistema Nacional de Agravos de Notificaçóes) between 2001 and 2008. We calculated the incidence of cutaneous leishmaniasis in each municipality. Leishmaniasis prevalence rate from 2001 to 2008 was higher in the cities of Nova Ubiratã (857.2 cases per 100,000 population), Feliz Natal $(834.5$ per 100,000) and Vera $(557.0$ per 100,000). The average incidence (334.9 per 100,000 population) recorded at the ERS-Sinop was 4.5 times higher than that recorded for the whole North Region of Mato Grosso. The disease was more severe for people between 20 and 49 years old, irrespective of gender. Incidence was higher in males (64.8\%). The number of cases recorded in the drought period was $60 \%$. In the region, part of the population of the study area is exposed to direct contact with the vector of Leishmania, while they are working in activities such as selective logging, farming and mining.
\end{abstract}

KEYWORDS: Epidemiology, Public Health, Prevalence, Amazonia.

\footnotetext{
1,2Secretaria Estadual de Saúde- Escritório Regional de Saúde de Sinop. Rua das Avencas, 2072 Centro. CEP: 78.556-087, Sinop, MT. E-mail: didinobres@hotmail.com; niseleite@hotmail.com

3 Universidade Federal de Mato Grosso. Núcleo de Estudos da Biodiversidade da Amazônia Mato-grossense, Instituto de Ciências Naturais, Humanas e Sociais. Sinop-MT. Av. Alexandre Ferronato, 1200. Setor Industrial. CEP: 78.557-267, Sinop, MT. E-mail:djmingo23@gmail.com
} 


\section{INTRODUÇÃO}

As Leishmanioses são antropozoonoses que constituem um grande problema de saúde pública, representando um complexo de doenças com importante espectro e diversidade epidemiológica (Vale e Furtado 2005), é considerada uma das cinco doenças infecto-parasitárias endêmicas de maior relevância mundial (Guerra et al. 2007). A Organização Mundial de Saúde (OMS) estima que 350 milhôes de pessoas estejam expostas ao risco de adoecer, com registro aproximado de dois milhôes de novos casos e diferentes formas clínicas ao ano. A leishmaniose tegumentar americana (LTA) é uma doença causada por protozoários do gênero Leishmania, acomete o homem na pele e/ou na mucosa, principalmente das vias aerodigestivas superiores, é considerada cosmopolita (atinge 88 países) e sua notificação é compulsória em apenas 30 países (Levine et al. 1980; Gontijo e Carvalho 2003). A transmissão da doença ocorre pela picada de fêmeas infectadas de dípteros da subfamília Phlebotominae, pertencentes aos gêneros Lutzomyia - no Novo Mundo, e Phlebotomus - no Velho Mundo (Walters 1993).

A confirmação no Brasil de formas leishmânicas em úlceras cutâneas e nasobucofaríngeas ocorreram no ano de 1909 por Lindenberg, ao encontrar o parasito em indivíduos que trabalhavam em áreas de desmatamentos na construção de rodovias no interior de Sáo Paulo (Vale e Furtado 2005). A forma mucosa da doença foi diagnosticada por Splendore em 1911 (Apud Ministério da Saúde 2010). Mesmo sendo registrada a ocorrência da doença no país há mais de 100 anos, os dados estimados de incidência da LTA no Brasil não são fidedignos (Basamo e Camargo 2004), porém sua prevalência vem aumentando, consideravelmente, em todas as regiōes (Silva et al. 1999; Monteiro et al. 2008) e com surtos epidêmicos (Dourado et al. 1989; Barros et al. 1985), principalmente, na região amazônica (Silva et al. 1999), considerada o mais importante foco de transmissão do país, com um coeficiente de detecçáo de 99,8/100.000 habitantes (Nunes et al. 1995). A partir da década de 80 , verificou-se um aumento no número de casos registrados, variando de 3.000 em 1980 para 35.747 em 1995. Os picos de transmissão são observados a cada cinco anos, a partir do ano de 1985, quando se consolida a implantaçâo das açóes de vigilância e controle da LTA no país. No período de 1985 a 2005, verificou-se uma média anual de 28.568 casos autóctones registrados (Ministério da Saúde 2010).

Mudanças no padrão de transmissão e expansão da LTA estão ocorrendo de forma endêmico-epidêmica em decorrência das modificaçóes ambientais, sociais e processos migratórios caracterizados pelo êxodo rural e abertura e colonizaçáo de novas áreas (Dourado et al. 1989; Leonardo e Rebêlo 2004; Vale e Furtado 2005). Além disso, mais evidências de ocorrência da doença em áreas de colonização antiga também foram registradas (Barros et al. 1985; Vale e Furtado 2005; Guerra et al. 2007). Nestas, discute-se a possível adaptação dos vetores e parasitas, causando dois padrôes epidemiológicos distintos, um relacionado à expansão das fronteiras agrícolas e outro ao crescimento de regiôes periurbanas, com possíveis adaptaçôes dos parasitos a reservatórios extra-silvestres.

Apesar de terem sido realizados vários estudos sobre a incidência da LTA em diversos estados brasileiros (Barros et al. 1985; Dourado et al. 1989; Silva et al. 1999; Aparício e Bitencourt 2004; Leonardo e Rebêlo 2004), no estado de Mato Grosso, principalmente na parte amazônica, os estudos ainda são escassos (Moura et al. 1999; Azevedo et al. 2002). A colonização desta regiáo foi reforçada no início da década de 70 do século $\mathrm{XX}$, suportada por projetos de reforma agrária e construção de rodovias do governo federal (Azevedo et al. 2002). Essas correntes de migração produziram grandes alteraçôes ambientais, causando novas associaçóes entre o homem e a natureza, originando novos focos de doenças entre elas a LTA e a malária (Azevedo et al. 2002).

$\mathrm{Na}$ regiáo norte do estado do Mato Grosso localiza-se Sinop, a maior cidade e pólo de desenvolvimento regional; ela é fruto de um dos projetos de colonização do Governo Federal. Mais de 15 cidades estão localizadas no entorno de Sinop. Nesta região, existem altos índices de desmatamento e as principais atividades econômicas são a exploração de madeira, agricultura e pecuária. $\mathrm{O}$ crescimento desordenado e os tipos de atividades desenvolvidas na regiāo têm aumentado o número de casos de leishmaniose tegumentar americana (LTA), que denota a importância epidemiológica de se realizar um estudo da doença no contexto amazônico e, em particular, na Amazônia Mato-grossense (Basamo e Camargo 2004). O presente estudo teve por objetivo analisar a prevalência da LTA nos municípios que integram a Microrregião Teles Pires - Escritório Regional de Saúde (ERS) Sinop.

\section{MATERIAL E MÉTODOS}

O estudo foi realizado no Escritório Regional de Saúde (ERS) de Sinop que está localizado na Microrregiāo Teles Pires, norte do Estado de Mato Grosso, abrangendo uma área de $1.050 .358,70 \mathrm{~km}^{2}$ (Figura 1). O município mais próximo fica a $35 \mathrm{~km}$ (Santa Carmem) e o mais distante a $290 \mathrm{~km}$ (Santa Rita do Trivelato) da sede do ERS.

O ERS de Sinop atende uma populaçáo estimada de 321.156 habitantes (Dados náo publicados da ERS-Sinop em fevereiro de 2011), representando $11 \%$ da população mato-grossense (IBGE 2010). O grande desenvolvimento econômico desta região é atribuído, principalmente, ao setor agropecuário e madeireiro tornando-se um grande atrativo para a migração da população de diversas regiôes. O período de colheita dos grãos vai de janeiro a maio e são determinantes para o aumento do número de casos da doença e de seu 


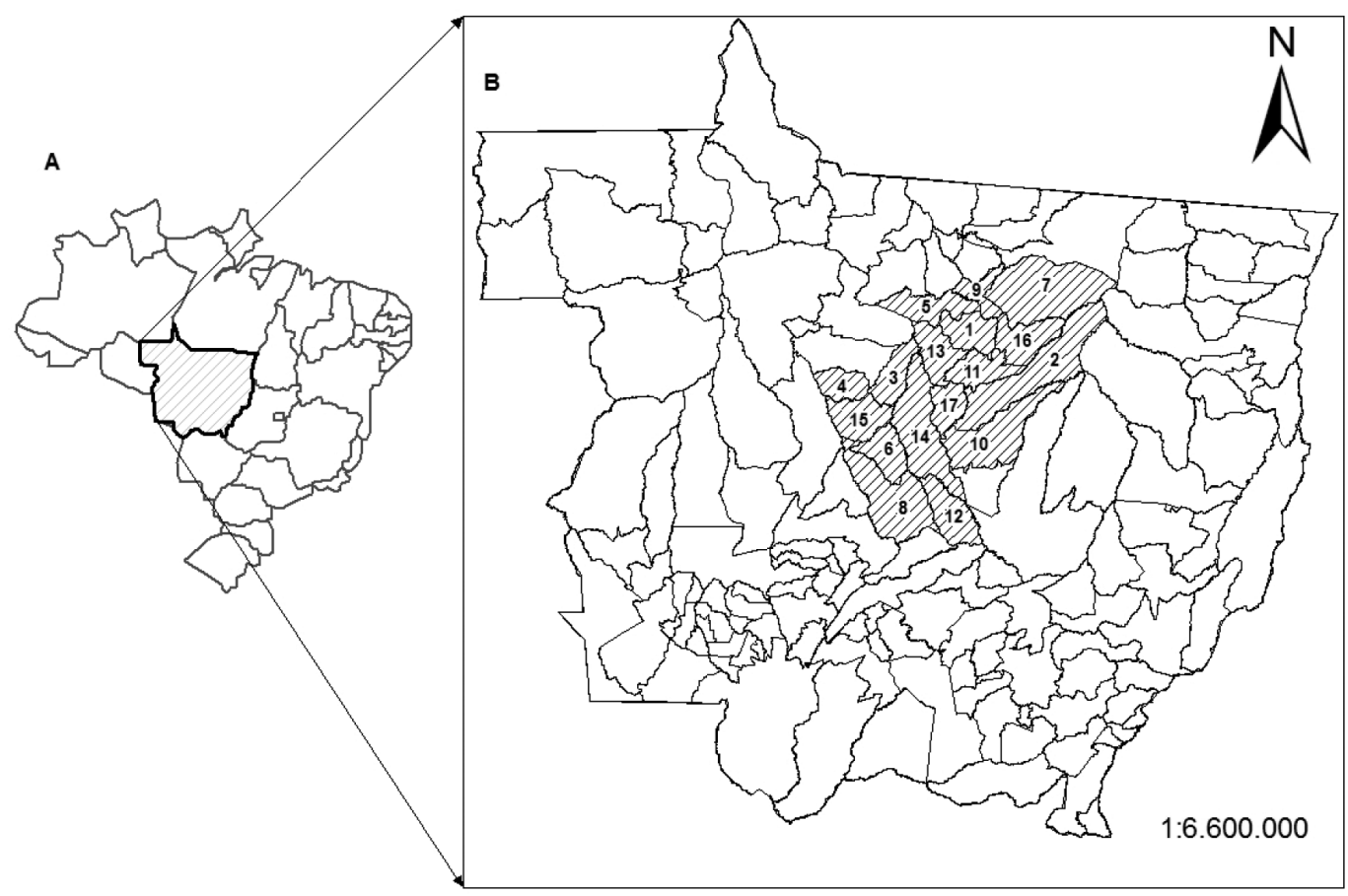

Figura 1 - Mapa do Brasil com a localização do Estado de Mato Grosso (A) e dos municípios pertencentes ao Escritório Regional de Saúde de Sinop (B): Cláudia (1), Feliz Natal (2), Ipiranga do Norte (3), Itanhangá (4), Itaúba (5), Lucas do Rio Verde (6), Marcelândia (7), Nova Santa helena (8), Nova Mutum (9), Nova Ubiratã (10), Santa Carmem (11), Santa Rita do Trivelato (12), Sinop (13), Sorriso (14), Tapurah (15), União do Sul (16) e Vera (17).

atendimento nos serviços de saúde dos municípios. O período chuvoso na região ocorre de outubro a abril e o período seco de maio a setembro.

Dos 17 municípios avaliados, 14 pertencem à jurisdição do ERS Sinop e três do ERS de Colider (Itaúba, Marcelândia e Nova Santa Helena). Os três municípios citados foram transferidos do ERS de Sinop para o ERS de Colider a partir de 2004, ano de sua criação e, apesar da desvinculação eles ainda são assessorados pelo ERS de Sinop em alguns programas, dentre eles o Sistema Nacional de Agravos de Notificação SINAN-NET. A população estimada para cada município em 2008, conforme IBGE registra-se a menor para Santa Rita do Trivelato (1.680 habitantes) e a maior para Sinop (95.193 habitantes) (Tabela 1).

A coleta de dados foi realizada por meio do Escritório Regional de Saúde, no Sistema Nacional de Agravos de Notificação - SINAN, entre 2001 a 2008. Os dados foram condensados no sistema de informação por representantes das secretarias municipais de saúde e enviados ao SINAN-NET, sendo este de responsabilidade da Vigilância Epidemiológica/ ERS. Os dados coletados no SINAN foram referentes aos municípios de residência, sexo, idade, grau de escolaridade, mês de ocorrência, forma da doença e casos de recidiva dos pacientes. Os dados de escolaridade foram definidos por meio do tempo de permanência escolar. Foram avaliadas diferenças entre o tempo de permanência escolar, e o número de casos notificados entre os sexos, idade dos pacientes e mês de ocorrência. A forma clínica foi definida em cutânea: presença de lesão localizada de forma única ou múltipla, e mucosa. A incidência média foi calculada pela média de casos notificados, multiplicado por 100.000 habitantes, e dividida pela média da população.

Para avaliar o número de casos de recidiva, foram consideradas as lesôes leishmanióticas reaparecidas em qualquer parte do corpo no período de até um ano após a cura clínica, informada na ficha de notificaçáo, sendo analisado o número de casos nos anos de estudo. Para avaliar a influência da sazonalidade na incidência de LTA, foram usados os dados de janeiro de 2001 a dezembro de 2008.

As análises com os dados de escolaridade (tempo de permanência escolar) foram realizadas apenas com os dados de 2001 a 2006, pois houve uma reestruturação e atualização de versão do sistema de informatização do SINAN em 2007, impossibilitando a extração dos dados e comprometendo assim a análise dos anos de 2007 e 2008.

\section{RESULTADOS}

No período de 2001 a 2008 o ERS/Sinop-MT registrou 7.374 casos de LTA (Tabela 1). O período de maior registro de casos foi entre 2002 e 2003, com uma leve diminuição nos anos posteriores. O município com maior número de 
Tabela 1 - Número de casos de leishmaniose tegumentar americana confirmados no período 2001 a 2008 nos municípios de abrangência do Escritório Regional de Saúde de Sinop, MT, retirados do SINAN com a incidência na população por 100.000 habitantes.

\begin{tabular}{lccccccccccccc}
\hline Município & 2001 & 2002 & 2003 & 2004 & 2005 & 2006 & 2007 & 2008 & CT & C & MP & Inc \\
\hline Claudia & 13 & 53 & 53 & 47 & 27 & 53 & 38 & 37 & 321 & 40,1 & 11210 & 357,9 \\
F Natal & 64 & 66 & 113 & 94 & 66 & 59 & 67 & 59 & 588 & 73,5 & 8808 & 834,5 \\
\hline I. Norte & 0 & 0 & 0 & 0 & 0 & 7 & 7 & 12 & 26 & 8,6 & 1431 & 605,6 \\
Itanhanga & 0 & 0 & 0 & 0 & 0 & 14 & 17 & 7 & 38 & 12,6 & 2240 & 565,4 \\
Itauba & 38 & 32 & 37 & 36 & 31 & 43 & 62 & 29 & 308 & 38,5 & 6035 & 637,9 \\
Lucas & 50 & 61 & 63 & 72 & 58 & 53 & 47 & 60 & 464 & 58,0 & 26083 & 222,4 \\
Marcelândia & 97 & 92 & 79 & 52 & 81 & 76 & 61 & 48 & 586 & 73,2 & 16737 & 437,7 \\
N S Helena & 0 & 18 & 15 & 9 & 7 & 6 & 5 & 7 & 67 & 8,4 & 3498 & 240,0 \\
N Mutum & 69 & 51 & 63 & 50 & 31 & 37 & 30 & 45 & 376 & 47,0 & 18190 & 258,4 \\
N Ubiratã & 33 & 85 & 68 & 63 & 73 & 72 & 51 & 41 & 486 & 61,0 & 7116 & 857,2 \\
\hline S R Trivelato & 0 & 9 & 10 & 12 & 12 & 12 & 10 & 7 & 72 & 9,0 & 1680 & 535,7 \\
Sta Carmem & 14 & 26 & 29 & 17 & 17 & 21 & 17 & 21 & 162 & 20,2 & 4160 & 485,5 \\
Sinop & 168 & 253 & 271 & 220 & 230 & 237 & 143 & 158 & 1680 & 210 & 95193 & 220,6 \\
Sorriso & 186 & 224 & 164 & 141 & 150 & 105 & 87 & 87 & 1144 & 143 & 46633 & 306,7 \\
\hline Tapurah & 79 & 53 & 53 & 73 & 75 & 27 & 39 & 40 & 439 & 54,9 & 10608 & 517,5 \\
U Sul & 13 & 12 & 33 & 24 & 25 & 18 & 15 & 11 & 151 & 18,9 & 5087 & 371,5 \\
\hline Vera & 64 & 67 & 84 & 56 & 63 & 45 & 47 & 40 & 466 & 58,2 & 10457 & 557,0 \\
\hline Total & 888 & 1102 & 1135 & 966 & 946 & 885 & 743 & 709 & 7374 & 921,7 & 275163 & 334,9 \\
\hline
\end{tabular}

CT = Casos totais. $C=$ Média de casos e MP = Média da população Inc= Incidência na população por 100.000 habitantes. Dados da população retirados do IBGE no ano de 2008.

casos registrados foi Sinop, seguido por Sorriso, Feliz Natal, Marcelândia e Nova Ubiratá (Tabela 1). A incidência média de casos notificados no período de 2001 a 2008 foi de 334,9 por 100.000 habitantes para o ERS/Sinop, sendo que a população do município de Nova Ubiratá ( 857,2 casos por 100.000 habitantes) apresentou maior risco de ser acometido pela doença (Tabela 1 ).

Em 2002 e 2003, ocorreram os maiores números de casos, concentrando-se entre os meses de maio a setembro, período de seca na região, correspondendo a $60 \%$ do total de casos entre os anos avaliados, nota-se uma ligeira diminuiçáo nos anos posteriores (Tabela 2). O número de casos confirmados foi maior no intervalo de 20 a 49 anos de idade, representando $64,78 \%$ casos (Tabela 3). O sexo masculino apresentou em todos os anos estudados uma maior ocorrência da doença, com 89\% de acometidos em relação ao sexo feminino (Tabela 3).

Indivíduos com tempo de permanência escolar entre quatro e sete anos adoeceram mais, e aqueles com 12 anos ou mais de escolaridade adoeceram menos, correspondendo a $50 \%$ e $3 \%$ do total, respectivamente (Tabela 4). A forma clínica com maior ocorrência foi a cutânea com 7.138 (96,80\%), seguido pela mucosa 217 (2,95\%). Foram registrados no ERS de Sinop 296 casos de recidiva, sendo o Município de Sorriso o mais acometido, com 79 casos $(26,6 \%)$.
Tabela 2 - Números mensais de casos notificados de leishmaniose tegumentar americana no período de 2001 a 2008 no Escritório Regional de Saúde de Sinop, no norte de Mato Grosso, considerando os períodos chuvosos (CH) e secos (SE).

\begin{tabular}{|c|c|c|c|c|c|c|c|c|c|c|}
\hline \multicolumn{11}{|c|}{ ANO } \\
\hline Mês & 2001 & 2002 & 2003 & 2004 & 2005 & 2006 & 2007 & 2008 & Total & Estação \\
\hline 空 & 45 & 63 & 65 & 39 & 39 & 55 & 22 & 37 & 365 & $\mathrm{CH}$ \\
\hline छें & 23 & 51 & 55 & 50 & 43 & 31 & 41 & 35 & 329 & $\mathrm{CH}$ \\
\hline$\sum^{\bar{\pi}}$ & 28 & 67 & 67 & 64 & 65 & 57 & 51 & 40 & 439 & $\mathrm{CH}$ \\
\hline 产 & 37 & 86 & 63 & 66 & 55 & 53 & 50 & 49 & 459 & $\mathrm{CH}$ \\
\hline$\sum^{\dot{\tilde{N}}}$ & 56 & 77 & 100 & 83 & 97 & 56 & 71 & 53 & 593 & SE \\
\hline 乌் & 79 & 96 & 88 & 92 & 89 & 63 & 75 & 79 & 661 & SE \\
\hline$\xi$ & 129 & 171 & 196 & 152 & 117 & 155 & 100 & 83 & 1103 & SE \\
\hline 定 & 196 & 150 & 172 & 141 & 156 & 143 & 119 & 128 & 1205 & SE \\
\hline ¿ & 85 & 129 & 127 & 129 & 121 & 106 & 80 & 86 & 863 & SE \\
\hline 芌 & 97 & 102 & 109 & 57 & 67 & 84 & 65 & 46 & 627 & $\mathrm{CH}$ \\
\hline oें & 66 & 66 & 53 & 52 & 66 & 49 & 44 & 51 & 447 & $\mathrm{CH}$ \\
\hline هั & 47 & 44 & 40 & 41 & 31 & 33 & 25 & 22 & 283 & $\mathrm{CH}$ \\
\hline 픙 & 888 & 1102 & 1135 & 966 & 946 & 885 & 743 & 709 & 7374 & \\
\hline
\end{tabular}


Tabela 3 - Distribuição de casos de leishmaniose tegumentar americana em relação ao sexo ( $\mathrm{M}=$ Masculino e $\mathrm{F}=$ Feminino) e faixa etária no período de 2001 a 2008 no Escritório Regional de Saúde de Sinop, no norte de Mato Grosso.

\begin{tabular}{|c|c|c|c|c|c|c|c|c|c|c|c|c|c|c|c|c|}
\hline \multirow[b]{3}{*}{ Idade } & \multicolumn{16}{|c|}{ Período amostrado } \\
\hline & \multicolumn{2}{|c|}{2001} & \multicolumn{2}{|c|}{2002} & \multicolumn{2}{|c|}{2003} & \multicolumn{2}{|c|}{2004} & \multicolumn{2}{|c|}{2005} & \multicolumn{2}{|c|}{2006} & \multicolumn{2}{|c|}{2007} & \multicolumn{2}{|c|}{2008} \\
\hline & M & $\mathrm{F}$ & $M$ & $\mathrm{~F}$ & M & $\mathrm{F}$ & M & $\mathrm{F}$ & M & $\mathrm{F}$ & M & $\mathrm{F}$ & M & $\mathrm{F}$ & M & $\mathrm{F}$ \\
\hline$<1$ & 2 & 0 & 9 & 0 & 2 & 1 & 0 & 1 & 3 & 0 & 6 & 4 & 4 & 0 & 8 & 2 \\
\hline 1 a 4 & 5 & 3 & 2 & 2 & 4 & 5 & 6 & 5 & 3 & 0 & 2 & 2 & 0 & 4 & 2 & 0 \\
\hline 5 a 9 & 8 & 5 & 8 & 9 & 10 & 3 & 11 & 6 & 4 & 7 & 7 & 9 & 5 & 4 & 1 & 1 \\
\hline 10 a 14 & 28 & 7 & 32 & 17 & 36 & 10 & 24 & 9 & 34 & 3 & 23 & 12 & 22 & 7 & 13 & 12 \\
\hline 15 a 19 & 93 & 12 & 95 & 15 & 111 & 9 & 77 & 5 & 94 & 7 & 58 & 4 & 61 & 6 & 54 & 3 \\
\hline 20 a 34 & 340 & 31 & 413 & 37 & 468 & 42 & 363 & 31 & 354 & 24 & 337 & 30 & 255 & 25 & 258 & 31 \\
\hline 35 a 49 & 226 & 28 & 293 & 30 & 289 & 27 & 292 & 25 & 269 & 30 & 232 & 38 & 198 & 31 & 190 & 22 \\
\hline 50 a 64 & 74 & 7 & 94 & 15 & 91 & 7 & 82 & 10 & 83 & 18 & 82 & 11 & 78 & 21 & 76 & 14 \\
\hline 65 a 79 & 14 & 4 & 26 & 3 & 15 & 5 & 14 & 1 & 10 & 2 & 13 & 1 & 17 & 3 & 19 & 3 \\
\hline $80+$ & 0 & 0 & 2 & 0 & 0 & 0 & 2 & 2 & 1 & 0 & 8 & 0 & 2 & 0 & 0 & 0 \\
\hline Total & 790 & 97 & 974 & 128 & 1026 & 109 & 871 & 95 & 855 & 91 & 768 & 111 & 640 & 101 & 621 & 88 \\
\hline TOTAL & \multicolumn{2}{|c|}{887} & \multicolumn{2}{|c|}{1102} & \multicolumn{2}{|c|}{1135} & \multicolumn{2}{|c|}{966} & \multicolumn{2}{|c|}{946} & \multicolumn{2}{|c|}{879} & \multicolumn{2}{|c|}{741} & \multicolumn{2}{|c|}{709} \\
\hline
\end{tabular}

Tabela 4 - Casos notificados no Escritório Regional de Saúde de SinopMT em relação ao tempo de permanência escolar em anos no período de 2001 a 2008 no norte de Mato Grosso. Os formulários ignorados (lg) e não preenchidos (Branco) também são apresentados.

Tempo de permanência escolar

\begin{tabular}{|c|c|c|c|c|c|c|c|c|}
\hline Ano & 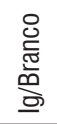 & $\begin{array}{l}\text { m } \\
\widetilde{\sigma} \\
-\end{array}$ & $\underset{\widetilde{\sigma}}{\sigma}$ & $\underset{\infty}{\mp}$ & $\stackrel{+}{\simeq}$ & $\bar{v}$ & $\begin{array}{l}\text { E } \\
\text { 竞 } \\
\text { 离 }\end{array}$ & Total \\
\hline 2001 & 44 & 35 & 627 & 73 & 9 & 19 & 81 & 888 \\
\hline 2002 & 26 & 273 & 532 & 145 & 44 & 21 & 61 & 1102 \\
\hline 2003 & 16 & 300 & 533 & 158 & 37 & 25 & 65 & 1135 \\
\hline 2004 & 9 & 217 & 470 & 166 & 22 & 26 & 56 & 966 \\
\hline 2005 & 12 & 193 & 436 & 185 & 40 & 15 & 65 & 946 \\
\hline 2006 & 14 & 178 & 357 & 215 & 41 & 25 & 55 & 885 \\
\hline Total & 121 & 1196 & 2955 & 942 & 193 & 131 & 383 & 5921 \\
\hline
\end{tabular}

\section{DISCUSSÃO}

O estudo realizado pelo Ministério da Saúde entre 1989 e 2008 sobre a ocorrência de LTA no Brasil revelou um aumento significativo no número de casos confirmados no CentroOeste, sendo considerada a segunda região de maior risco no país (média de 37,5 por 100.000 habitantes), estando acima do Nordeste (média de 18 por 100.000 habitantes), atrás apenas da regiáo Norte (média de 85,4 por 100.000 habitantes) (Ministério da Saúde 2010). Em Mato Grosso, a LTA é uma das mais importantes afecçôes dermatológicas, devido à sua alta incidência e relevante endemicidade, colocando o estado em segundo lugar em nível nacional de detecção de casos
(Ministério da Saúde 2012a). Esse fato, acarreta consequências negativas no âmbito social e econômico. Uma vez que, em geral a leishmaniose é considerada uma doença ocupacional. No ERS de Sinop a ocorrência representa, quase 4,5 vezes a mais que a média da regiâo norte no país (Ministério da Saúde 2012b). Provavelmente, essa alta incidência está relacionada com as atividades laborais desenvolvidas na regiáo como atividades garimpeiras, exploração de madeiras, agricultura, desmatamento para a implantação de pastagens, grande número de assentamentos agrários próximos a áreas de florestas e condiçóes climáticas favoráveis para o desenvolvimento do vetor. Resultados similares foram registrados por Naiff-Júnior et al. (2009) em assentamentos rurais na rodovia AM-010, no estado do Amazonas, Sampaio e Paula (1999) no Distrito Federal e Azevedo et al. (2002) no município de Peixoto de Azevedo no norte do Estado do Mato Grosso.

No ERS de Sinop, nos anos de 2002 e 2003 houve um aumento considerável no número de casos registrados de LTA que podem estar relacionados diretamente com os processos de ocupação desordenada ou às chamadas invasóes, atividades comuns nesta regiáo, durante esse período (E. Nobres, observação pessoal; SINAN-NET, Dados não publicados). Existe uma forte associação entre trabalho agrícola e LTA principalmente em áreas amazônicas (Dourado et al. 1989; Guerra et al. 2006). O resultado desse estudo é semelhante ao encontrado por Chagas et al. (2006) que relataram aumento no número de notificaçôes de LTA em municípios pertencente ao estado do Amazonas no ano de 2003, com uma subsequente diminuiçáo nos anos posteriores, devido aos impactos direto 
das medidas de controle sanitário, como as aplicadas na vila de Pitinga, Amazonas.

Indivíduos do sexo masculino, na faixa etária entre 20 e 49 anos e com tempo de permanência escolar curto foram os mais acometidos pela LTA nos municípios do ERS/Sinop. Isso demonstra que os acometidos estáo em idade produtiva e provavelmente trabalhando em locais cuja veiculação da doença está presente. Esta é uma característica da regiâo aonde o setor do agronegócio (indústrias madeireiras e agropecuárias) contrata homens, principalmente aqueles com baixa escolaridade para o desenvolvimento dessas atividades. A LTA comumente é observada com mais intensidade em áreas onde o processo de colonização é recente, colocando preferencialmente homens jovens em contato com os elos da cadeia epidemiológica da doença (Silva et al. 1999; Azevedo et al. 2002, Guerra et al. 2006). Esses resultados corroboram com os obtidos por Chagas et al. (2006), Silva e Cunha (2007) e Naiff-Júnior et al. (2009) que encontraram maiores incidência entre homens na faixa etária de 20 e 39 anos. verificaram que os pacientes com LTA da regiáo do Vale do Arinos, Mato Grosso, tiveram pouco tempo de permanência na escola (entre quatro e sete anos de estudo), corroborando com os dados encontrados na regional de Sinop. Isso ocorre porque a maioria das atividades desenvolvidas naquela regiấo é decorrente do extrativismo de madeira, garimpo e/ou atividades agrícolas.

A maior ocorrência de LTA foi detectada na estação seca (4425), pois nesse período intensificam-se as atividades agropastoris dentre elas: $\mathrm{O}$ desmatamento, o preparo de solo para plantio, a extração de minerais e a extração seletiva de madeira, essas atividades propiciam uma exposição mais constante ao vetor. Resultado semelhante foi relatado por Dourado et al. (1989) em Lençóis, no Estado da Bahia, cujas atividades foram similares às encontradas na região do ERS/ Sinop. No entanto, Chagas et al. (2006) e Naiff-Júnior et al. (2009) encontraram uma maior ocorrência da doença no período chuvoso, relacionando essa ocorrência à maior abundância do vetor para a Amazônia Central brasileira. A diferença encontrada neste estudo em relaçáo aos trabalhos de Chagas et al. (2006) e Naiff-Júnior et al. (2009) podem ser explicadas pela extensão do período chuvoso, que no centro-norte do estado do Amazonas, onde foram realizados esses estudos, é mais longo que no sul da Amazônia (região estudada). Desse modo, as atividades de madeireiras e de mineração são reduzidas no período chuvoso, diminuindo o número de casos de LTA.

O município de Sorriso apresentou o maior número de recidivas $(76,26 \%)$. Este fato pode estar relacionado com o aumento das invasóes de terras no município e a criação de vários assentamentos rurais, pois grande parte das casas em assentamentos populacionais recentes são construídas a menos de $100 \mathrm{~m}$ da borda da floresta deixando os indivíduos expostos aos vetores, além da dificuldade de acesso aos serviços de saúde para o tratamento da doença, o que propicia o aumento da frequência da LTA por recidiva, conforme o registro de Passos et al. (2001).

Quanto às formas clínicas, observou-se que a predominância de casos se dá na forma cutânea com $96,80 \%$ dos casos. Os resultados obtidos são semelhantes aos encontrados por Thies e Sirena (2007) com 84,12\% para lesóes cutâneas na região do Vale do Arinos, norte de Mato Grosso. Neste estudo, verificouse que uma parte significativa da população da regiáo estudada está exposta ao contato direto com o vetor da Leishmania, principalmente devido às características da regiáo por serem semelhantes às da regiáo Norte do Brasil.

Os resultados desse estudo apontam que a expansão territorial do desmatamento, das atividades agrícolas e madeireiras ampliam a incidência de LTA e a sua dinâmica de transmissão conforme postulado por Martins et al. (2004). Outros fatores importantes nessa dinâmica são as atividades comerciais de cada regiáo, que nas áreas de concentração deste estudo sáo desempenhadas por meio de atividades madeireiras e agropastoris, que afetam uma parte significativa de indivíduos do sexo masculino, os quais são mais acometidos pela LTA. Entretanto, novos estudos deverão ser realizados em outros Escritórios Regionais de Saúde na Amazônia, principalmente nos Estado do Acre, Amazonas, Mato Grosso, Pará e Rondônia para ter um diagnóstico mais realístico da distribuição de casos de LTA na Amazônia, compará-los com outras regiôes do Brasil e verificar as profissóes dos pacientes afetados pela LTA.

\section{AGRADECIMENTOS}

Agradecimentos: Ao Escritório Regional de Saúde de Sinop pelo fornecimento dos dados usados nesse artigo. Anne T. D'Heursel Baldisseri pela revisão do abstract. Lindiomar Martins dos Santos pela revisão da língua portuguesa.

\section{BIBLIOGRAFIA CITADA}

Aparício, C.; Bitencourt, M.D. 2004. Modelagem espacial de zonas de risco da leishmaniose tegumentar americana. Revista de Saúde Pública, 38: 511-516.

Azevedo, A.C.R.; Souza, N.A.; Meneses, C.R.V.; Costa, W.A.; Costa, S.M.; Lima, J.B.; Rangel, E.F. 2002. Ecology of Sand Flies (Diptera: Psychodidae: Phlebotominae) in the North of the State of Mato Grosso, Brazil. Memórias do Instituto Oswaldo Cruz, 97: 459-464.

Barros, G.C.; Sessa, P.A.; Mattos, E.A.; Carias, V.R.D.; Mayrink, W.; Alencar, J.T.A; Falqueto, A.; Jesus, A. 1985. Foco de leishmaniose tegumentar americana nos municípios de Viana e Cariacica, Estado do Espírito Santo. Brasil. Revista de Saúde Pública, 19: 146-153. 
Basamo, A.S; Camargo, L.M.A. 2004. Leishmaniose tegumentar americana: histórico, epidemiologia e perspectivas de controle. Revista Brasileira de Epidemiologia, 7: 328-337.

Chagas, A.C.; Pessoa, F.A.C.; Medeiros, J.F.; Py-Daniel, V.; Mesquita, E.C.; Balestrassi, D.A. 2006. American Cutaneous Leishmaniasis (ACL) in a mining village - Pitinga, Amazonas, Brazil. Revista Brasileira de Epidemiologia, 9: 186-92 (in Portuguese, with abstract in English).

Dourado, M.I.C; Noronha, C.V.; Alcântara, N.; Ichihara, M.Y.T.; Loureiro, S. 1989. Epidemiologia da leishmaniose tegumentar americana e suas relações com a lavoura e o garimpo, em localidade do Estado da Bahia. Brasil. Revista de Saúde Pública, 23: 2-8.

Gontijo, B.; Carvalho, M.R.L. 2003. Leishmaniose tegumentar americana. Revista da Sociedade Brasileira de Medicina Tropical, 36: 71-80.

Guerra, J.A.O.; Ribeiro, J.A.S.; Coelho, L.I.A.R.C.; Barbosa, M.G.V.; Paes, M.G. 2006. Epidemiologia da Leishmaniose Tegumentar na comunidade São João, Manaus, Amazonas, Brasil. Cadernos de Saúde Pública, 22: 2319-2327.

Guerra, J.A.O.; Barbosa, M.G.V.; Loureiro, A.C.S.P.; Coelho, C.P.; Rosa, G.G.; Coelho, L.I.A.R.C. 2007. American tegumentary leishmaniasis in children: epidemiological aspects of cases treated in Manaus, Amazonas, Brazil. Cadernos de Saúde Pública, 23: 2215-2223 (in Portuguese, with abstract in English).

IBGE. 2010. Censo Demográfico. http://www.ibge.gov.br/home/ estatistica/populacao/censo2010/default.shtm. Acesso em 22/11/2011.

Leonardo, F.S.; Rebêlo, J.M.M. 2004. A periurbanização de Lutzomyia whitmani em área de foco de leishmaniose cutânea, no Estado do Maranháo. Brasil. Revista da Sociedade Brasileira de Medicina Tropical, 37: 282-284.

Levine, N.D.; Corliss, J.O.; Cox, F.E.G.; Deroux, G.; Grain, J.; Ronigberg, B.M.; Lieedale, G.F.; Leoeblich, A.R.; Lom, J.; Lynn, D.; Merinfeld, EG.; Page, F.C.; Poljansky, G.; Sprague, V.; Vávra, J. Wallace, F.G.A. 1980. Newly revised classification of the PROTOZOA. Journal of Protozoology, 27: 37-58.

Martins, L.M.; Rebêlo, J.M.M.; Santos, M.F.V.; Costa, J.M.L.; Silva, A.R.; Ferreira, L.A. 2004. Eco-epidemiology of cutaneous leishmaniasis in Buriticupu, Amazon region of Maranhão State, Brazil, 1996-1998. Cadernos de Saúde Pública, 20: 735-743 (in Portuguese, with abstract in English).

Monteiro, W.M.; Neitzke, H.C.; Lonardoni, M.V.C.; Silveira, T.G.V.; Ferreira, M.E.M.C.; Teodoro, U. 2008. Geographic distribution and epidemiological features of American tegumentary leishmaniasis in old rural settlements in Paraná State, Southern Brazil. Cadernos de Saúde Pública, 24: 1291-1303

Moura, S.T.; Fernandes, C.G.N.; Pandolpho, V.C.; Silva, R.R. 1999. Diagnóstico de leishmaniose canina na área urbana do município de Cuiabá, Estado de Mato Grosso, Brasil. Brazilian Journal of Veterinary Research and Animal Science, 36: 1-5.

Ministério da Saúde. 2010. Manual de Vigilância da Leishmaniose Tegumentar Americana. 2a edição Atualizada. Editora do Ministério da Saúde, Brasília. 180p.
Ministério da Saúde. 2012a. Leishmaniose Tegumentar Americana. Guia de Vigilância Epidemiológica, Caderno 11. 1-30. http:// portal.saude.gov.br/portal/arquivos/pdf/gve_7ed_web_atual_lta. pdf. Acesso em 05/04/2012.

Ministério da Saúde. 2012b. Coeficiente de detecção de casos de Leishmaniose Tegumentar Americana por 100.000 habitantes. Brasil, Grandes Regiōes e Unidades Federadas. 1990 a 2010. http://portal.saude.gov.br/portal/arquivos/pdf/ lta_deteccao_08_09_11.pdf. Acesso em 05/04/2012.

Naiff-Júnior, R.D.; Pinheiro, F.G.; Naiff, M.F.; Souza, I.S.; Castro, L.M.; Menezes, M.P.; Franco, A.M.R. 2009. Estudo de uma série de casos de Leishmaniose Tegumentar Americana no município de Rio Preto da Eva, Amazonas, Brasil. Revista de Patologia Tropical, 38: 103-104.

Nunes, V.L.B.; Dorval, M.E.M.C.; Oshiro, E.T.; Noguchi, R.C.; Arão, L.B.; Hans-Filho, G. 1995. Estudo Epidemiológico sobre Leishmaniose Tegumentar (LT) no município de Corguinho, Mato Grosso do Sul - Estudos na População Humana. Revista da Sociedade Brasileira de Medicina Tropical, 28: 185-193.

Passos, V.M.A.; Barreto, S.M.; Romanha, A.J.; Krettli, A.U.; Volpini, A.C.; Gontijo, C.M.F.; Falcão, A.L.; Lima-Costa, M.F. 2001. Leishmaniose Tegumentar na Região Metropolitana de Belo Horizonte: Aspectos clínicos, laboratoriais, terapêuticos e Evolutivos (1989 - 1995). Revista da Sociedade Brasileira de Medicina Tropical, 34: 5-12.

Sampaio, R.N.R.; Paula, C.D.R. 1999. Leishmaniose Tegumentar Americana no Distrito Federal. Revista da Sociedade Brasileira de Medicina Tropical, 32: 523-528.

Silva, L.M.R.; Cunha, P.R. 2007. Urbanization of American Cutaneous Leishmaniasis in Campinas - Sao Paulo (SP) and region: problems and challenges. Revista brasileira de Dermatologia, 82: 515-519

Silva, N.S.; Viana, A.B.; Cordeiro, J.A; Cavasini, C.E.; 1999. Leishmaniose tegumentar americana no Estado do Acre. Revista de Saúde Pública, 33: 554-559.

Thies, F.S.; Sirena, S.R.C. 2007. Perfil epidemiológico dos pacientes acometidos por leishmaniose tegumentar americana (LTA) na Região do Vale do Arinos no ano de 2006. 18 Encontro de Biólogos do CRBio-01, 31 de março a 03 de abril de 2007, Cuiabá, MT.179p.

Vale, E.C.S.; Furtado, T. 2005. Tegumentary leishmaniasis in Brazil: a historical review related to the origin, expansion and etiology. Anais Brasileiros de Dermatologia, 80: 421-428

Walters, L.L. 1993. Leishmania differentiation in natural and unnatural sand fly hosts. Journal Eukaryotic Microbiology, 40: 196-206.

Recebido em: 14/02/2012

Aceito em: 16/04/2012 
